\title{
Atomistic Simulation of Atomic Force Microscopy Imaging of Hydration Layers on Calcite, Dolomite, and Magnesite Surfaces
}

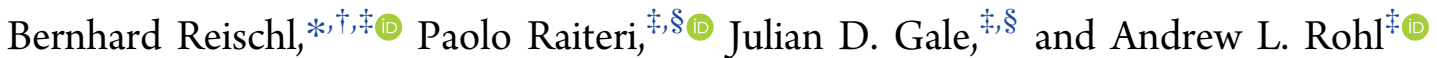 \\ ${ }^{\dagger}$ Institute for Atmospheric and Earth System Research/Physics, University of Helsinki, P.O. Box 64, Helsinki, FI-00014, Finland \\ ${ }^{\ddagger}$ Curtin Institute for Computation and School of Molecular and Life Sciences, and ${ }^{\S}$ The Institute for Geoscience Research, Curtin \\ University, P.O. Box U1987, Perth, Western Australia 6845, Australia
}

Supporting Information

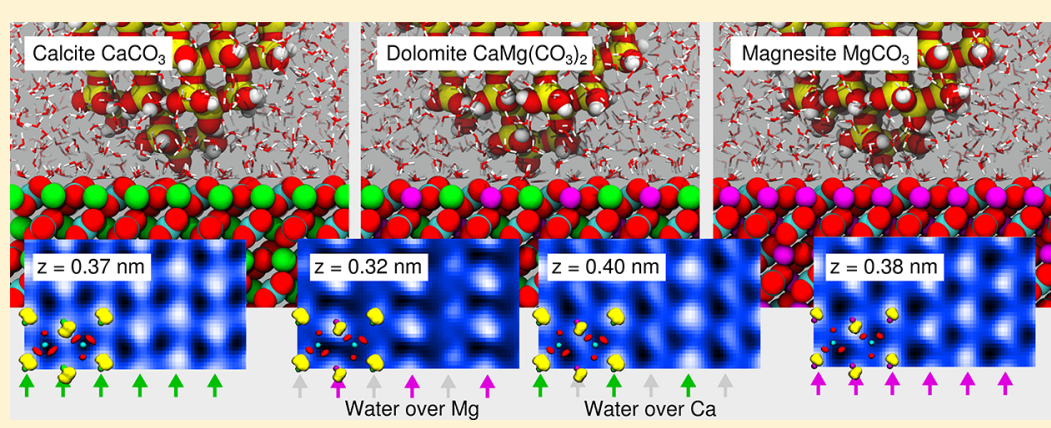

ABSTRACT: Advances in atomic force microscopy (AFM) in water have enabled the study of hydration layer structures on crystal surfaces, and in a recent study on dolomite $\left(\mathrm{CaMg}\left(\mathrm{CO}_{3}\right)_{2}\right)$, chemical sensitivity was demonstrated by observing significant differences in force-distance curves over the calcium and magnesium ions in the surface. Here, we present atomistic molecular dynamics simulations of a hydration layer structure and dynamics on the (10 $\overline{1} 4)$ surfaces of dolomite, calcite $\left(\mathrm{CaCO}_{3}\right)$, and magnesite $\left(\mathrm{MgCO}_{3}\right)$, as well as simulations of AFM imaging on these three surfaces with a model silica tip. Our results confirm that it should be possible to distinguish between water molecules coordinating the calcium and magnesium ions in dolomite, and the details gleaned from the atomistic simulations enable us to clarify the underlying imaging mechanism in the AFM experiments.

\section{INTRODUCTION}

The atomic force microscope can be operated in environments ranging from ultrahigh vacuum to ambient air to liquids. In recent years, great improvements have been made in experimental methodology, which not only made it possible to obtain atomic resolution $2 \mathrm{D}$ images of surfaces in solution, ${ }^{1-3}$ but also 3D data sets that allow the study of hydration layers at the solid-liquid interface. ${ }^{4-7}$ At the same time, efforts have been made to establish a theoretical understanding of the interactions between the AFM tip, water, and sample surface using both atomistic models including an explicit model of the AFM tip, ${ }^{8-10}$ as well as simpler models, focusing on the equilibrium hydration layer structure. $^{11,12}$ A combined analysis of 3D frequency modulation AFM (FM-AFM) data and atomistic simulation with a model AFM tip concluded that the "forest of peaks" seen in force-distance curves at the calcite-water interface are indeed a signature of the equilibrium hydration layer structure. ${ }^{13} \mathrm{~A}$ computational study on AFM imaging of surfaces with point defects in solution found that a sharp, hydroxylated silicon AFM tip should be able to resolve a magnesium substitution in the surface of calcite $\left(\mathrm{CaCO}_{3}\right)$, despite this being a chargeneutral defect, ${ }^{14}$ consistent with recent experimental observations. $^{15}$
Dolomite $\left(\mathrm{CaMg}\left(\mathrm{CO}_{3}\right)_{2}\right)$ has a similar crystal structure to calcite, but exhibits alternating rows of $\mathrm{Ca}$ and $\mathrm{Mg}$ ions along the $[42 \overline{1}]$ direction in the $(10 \overline{1} 4)$ surface, which have been atomically resolved by bimodal AFM imaging in ultrahigh vacuum. ${ }^{16}$ Although an earlier AFM study of dolomite in water could not distinguish between the $\mathrm{Ca}$ and $\mathrm{Mg}$ sublattice, ${ }^{17}$ in a recent paper Söngen and co-workers have demonstrated that this "chemical identification" could be achieved, ${ }^{18}$ based on statistically significant differences in the force-distance curves over the presumed $\mathrm{Ca}$ and $\mathrm{Mg}$ sublattices. These variations were attributed to the different hydration layer structures over the two distinct cation sites, observed in molecular dynamics (MD) simulations of the dolomite-water interface in the absence of the AFM tip. In order to investigate the origin of the contrast seen in the experiment-and find out whether theory can support the claim of chemical sensitivity-we have carried out large-scale atomistic simulations of the AFM imaging of calcite, dolomite, and magnesite surfaces in water, with a model silicon AFM tip. The effective tip-surface interactions obtained from the atomistic simulations were then

Received: January 30, 2019

Revised: $\quad$ May 27, 2019

Published: May 27, 2019 
used to simulate FM-AFM data using the virtual AFM methodology.

\section{METHODS}

MD Setup. All atomistic simulations have been performed using the large-scale atomic/molecular massively parallel simulator (LAMMPS) MD code, ${ }^{19}$ with a velocity Verlet integrator and a time step of 1 fs. An NVT ensemble of temperature $300 \mathrm{~K}$ was realized using a Nosé-Hoover chain thermostat of length 5 with a time constant of 1 ps. For the preliminary study of equilibrium properties of the aqueous interfaces of calcite $\left(\mathrm{CaCO}_{3}\right)$, dolomite $\left(\mathrm{CaMg}\left(\mathrm{CO}_{3}\right)_{2}\right)$, and magnesite $\left(\mathrm{MgCO}_{3}\right)$, we have carried out unbiased $\mathrm{MD}$ simulations to obtain a $40 \mathrm{~ns}$ trajectory for calcite, and longer 200 ns trajectories for dolomite and magnesite, given the slower dynamics of water in proximity to magnesium. ${ }^{20,21}$ The three systems consisted of an eight-layer thick slab of the mineral with heights of approximately $2.4,2.3$, and $2.2 \mathrm{~nm}$ for calcite, dolomite, and magnesite, respectively, in contact with water, exposing the (1014) cleavage plane perpendicular to the $z$ direction of the orthorhombic simulation box. In the plane of the surface ( $x$ and $y$ directions), the system was constructed using $5 \times 8$ surface unit cells, respectively, corresponding to simulation boxes measuring $4.044 \times 3.948 \times 9.821,3.846 \times$ $3.835 \times 8.867$, and $3.673 \times 3.692 \times 8.814 \mathrm{~nm}^{3}$ for calcite, dolomite, and magnesite. The height of the box was chosen to be large enough to ensure bulk-like water between the periodic images of the interfaces and was adjusted to yield the correct bulk water density. The $\mathrm{Ca}$ and/or $\mathrm{Mg}$ ions as well as the carbon atoms in carbonate in the fourth layer from the bottom were kept at fixed positions, in order to immobilize the mineral slab in the frame of reference of the simulation box. Silicon or silicon nitride AFM tips typically used for imaging in water are covered in a native oxide layer and will further react with water to form silanol groups $(\mathrm{Si}-\mathrm{OH})$ at the surface. We have therefore chosen to model the AFM tip apex using a chargeneutral silicon dioxide cluster, with all dangling bonds converted into silanol groups, and exposing a sharp termination in a single $\mathrm{OH}$ group toward the sample surface. A system consisting of a dolomite surface, silica tip model, and water is illustrated in Figure 1; an analogous setup was used for calcite and magnesite systems.

Force Field. The atomistic interactions of the three minerals and water were described by the force field of Raiteri et al., ${ }^{22}$ which uses the SPC/Fw potential for water, ${ }^{23}$ and reproduces the structural and thermodynamic properties of the carbonate minerals as well as the aqueous ions, and is therefore believed to accurately model the solid-liquid interfaces. $\mathrm{ClayFF}^{24}$ was used to describe interactions within the silica tip as well as between tip atoms and water molecules. Because of the absence of $\mathrm{Si}-\mathrm{O}-\mathrm{Si}$ angle terms in ClayFF, the cubic cristobalite structure is favored over $\alpha$-quartz at low pressure. As the exact structure of the amorphous oxide layer at the surface of a real AFM tip is unknown, we have chosen to build the tip model from cristobalite, which has a density similar to amorphous silica. ClayFF describes the hydration layers of $\mathrm{SiO}_{2}$ surfaces reasonably well, compared to other empirical potentials, benchmarked against density functional theory calculations. ${ }^{25,26}$ The missing interaction parameters between tip and surface atoms listed in Table 1 were either constructed from combination rules, or assigned based on similarities with atom types available in the force field, as specified in the Supporting Information.

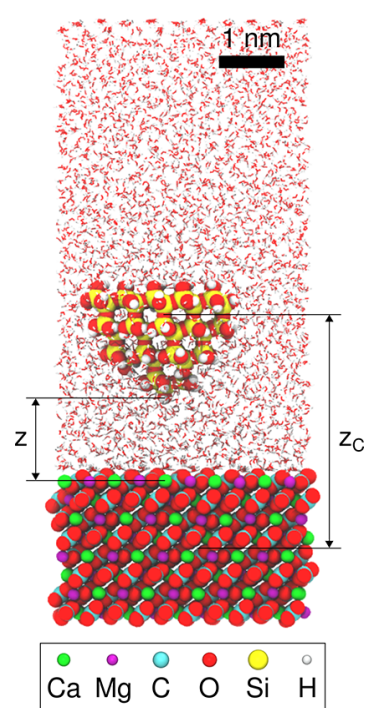

Figure 1. AFM simulation setup: snapshot of the MD trajectory of the simulation of AFM imaging of a dolomite (1014) surface in water with a silicon tip model. Calcium, magnesium, carbon, oxygen, silicon, and hydrogen atoms in the tip and surface are colored in green, purple, cyan, red, yellow, and white, respectively. Water molecules are shown as red and white stick models. The definitions of the tipsurface distance $\mathrm{CV} z_{\mathrm{C}}$, used in the free energy calculations, and the tip apex to surface layer distance $z$, are indicated by black arrows.

Table 1. Additional Tip-Surface Interaction Parameters for Calcium (Ca) and Magnesium (Mg) Ions, Carbonate Carbon (C), and Oxygen (Oc) from Raiteri et al. ${ }^{22}$ and Silicon ( $\mathrm{Si}$ ), Bridging Oxygen (Ob), Hydroxyl Oxygen (Oh), Hydroxyl Hydrogen (Ho) from ClayFF ${ }^{24 a}$

\begin{tabular}{|c|c|c|c|}
\hline \multicolumn{4}{|c|}{ Lennard-Jones, $U_{i j}(r)=4 \varepsilon\left[(\sigma / r)^{12}-(\sigma / r)^{6}\right]$} \\
\hline$i$ & $j$ & $\varepsilon(\mathrm{eV})$ & $\sigma(\AA)$ \\
\hline $\mathrm{Ca}$ & $\mathrm{Si}$ & $3.269000 \times 10^{-6}$ & 3.418268 \\
\hline $\mathrm{Ca}$ & $\mathrm{Ob}$ & $9.499140 \times 10^{-4}$ & 3.350025 \\
\hline $\mathrm{Mg}$ & $\mathrm{Si}$ & $3.913000 \times 10^{-6}$ & 2.888268 \\
\hline $\mathrm{Mg}$ & $\mathrm{Ob}$ & $1.136899 \times 10^{-3}$ & 2.820025 \\
\hline Oc & $\mathrm{Si}$ & $2.319120 \times 10^{-5}$ & 3.233784 \\
\hline Oc & $\mathrm{Ob}$ & $6.738784 \times 10^{-3}$ & 3.165541 \\
\hline $\mathrm{Oc}$ & $\mathrm{Oh}$ & $6.738784 \times 10^{-3}$ & 3.165541 \\
\hline \multicolumn{4}{|c|}{ Lennard-Jones repulsion, $U_{i j}(r)=A / r^{12}$} \\
\hline$i$ & \multicolumn{2}{|r|}{ j } & $A\left(\mathrm{eV} \cdot \AA^{12}\right)$ \\
\hline Oc & \multicolumn{2}{|r|}{ Ho } & 34.0 \\
\hline \multicolumn{4}{|c|}{ Buckingham repulsion, $U_{i j}(r)=A \exp (-r / \rho)$} \\
\hline$i$ & $j$ & $A(\mathrm{eV})$ & $\rho(\AA)$ \\
\hline $\mathrm{Ca}$ & Oh & 2885.525464 & 0.271511 \\
\hline $\mathrm{Mg}$ & $\mathrm{Oh}$ & 3854.970524 & 0.236073 \\
\hline $\mathrm{C}$ & $\mathrm{Ob}$ & 61.974708 & 0.570000 \\
\hline $\mathrm{C}$ & $\mathrm{Oh}$ & 79.926674 & 0.570000 \\
\hline
\end{tabular}

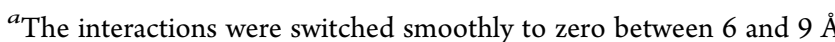
using the tapering function described in ref 22 .

Free Energy Calculations. For the AFM simulation, we used the same procedure as in previous work: ${ }^{27}$ the silica AFM tip model was introduced in the three simulation cells described above and the height of the simulation boxes was adjusted to yield the correct bulk water density away from the tip and surface. The new cell lengths along $z$ were 9.800, 9.300, and $8.596 \mathrm{~nm}$ for calcite, dolomite, and magnesite, respectively, sufficient to ensure bulk water between the top of the tip and 
the periodic image of the surface slab even at the largest tipsurface separation distances. The simulation boxes were also large enough along $x$ and $y$ to avoid spurious interactions between periodic images of the tip model, which measured approximately $2.2,2.3$, and $2.7 \mathrm{~nm}$ along the $x, y$, and $z$ directions, respectively. Using the PLUMED plug-in ${ }^{28}$ for LAMMPS, we performed umbrella sampling to calculate the free energy as a function of a tip-surface separation collective variable $(\mathrm{CV})$ for $8 \times 4$ lateral positions over each crystal's surface unit cell. The $\mathrm{CV}$ was defined as the $z$ component of the center of mass distance between the $\mathrm{Si}$ atoms of the upper part of the tip model and the entire mineral slab. The lateral position of these $\mathrm{Si}$ atoms was constrained by a harmonic potential with a spring constant of $100 \mathrm{eV} / \AA^{2}$, acting along the $x$ and $y$ directions. The starting configurations for the umbrella windows were obtained from preliminary steered MD simulations, and initially the CV range was sampled by 32 umbrellas, separated by $0.5 \AA$ along the $\mathrm{CV}$ and using a harmonic umbrella potential with a spring constant of $0.5 \mathrm{eV} /$ $\AA^{2}$. Where necessary, additional umbrellas at intermediate positions, or with stiffer spring constants of up to $5.0 \mathrm{eV} / \AA^{2}$, were introduced until the overlap in the distribution of the $\mathrm{CV}$ between neighboring windows was deemed satisfactory. The unbiased free energy profiles were obtained through selfconsistent histogram reweighting, using the weighted histogram analysis method code. 29

For the sake of comparability between the three systems, we have converted the tip-surface distance $\mathrm{CVs}, z_{\mathrm{C}}$, used in the free energy calculations, into tip apex to surface distances $z$, by computing the average differences between $z_{\mathrm{C}}$ and the distance along $z$ between the silanol oxygen atom at the tip apex, and the center of mass of the $\mathrm{Ca}$ and/or $\mathrm{Mg}$ atoms in the surface layer, for large tip-surface separation distances. This means that the value of $z$ does not take into account deformation of the tip apex in the strongly repulsive near-contact range, and a nondeformed tip "in contact" with, for example, the calcite surface would correspond to values of $z$ close to the distance of the first maximum in the radial distribution function between silanol oxygen and the $\mathrm{Ca}^{2+}$ ion, that is $z \approx 0.24 \mathrm{~nm}$. The AFM simulation setup and the definitions of $z_{\mathrm{C}}$ and $z$ are illustrated in Figure 1.

Virtual AFM. Simulated FM-AFM images were obtained with the Python Virtual AFM code (PyVAFM), ${ }^{30}$ using cantilever parameters similar to the AFM experiments on calcite and dolomite performed by Söngen et al. ${ }^{18}$ (force constant $k=40 \mathrm{~N} / \mathrm{m}$, quality factor $Q=8$, amplitude $A_{0}=0.08$ $\mathrm{nm}$, and resonant frequency $f_{0}=150 \mathrm{kHz}$ ). For each of the three systems, the PyVAFM was set up to record 2D constant height frequency modulation maps every $0.02 \mathrm{~nm}$ along $z$, with the fast scanning direction along $x$ and the slow direction along $y$. The effective 3D force field of tip-sample interaction, $f_{z}(x, y, z)$, was obtained from the numerical derivatives of the free energy profiles obtained in the atomistic simulation, $f_{z}(x, y, z)=-\mathrm{d} F(x, y ; z) / \mathrm{d} z$, after performing a running average over three consecutive data points, separated by $0.01 \mathrm{~nm}$. Using a cubic spline, the force curves were laterally interpolated from the original $8 \times 4$ grid to obtain a $16 \times 8$ grid on each surface unit cell along $x$ and $y$ directions, respectively, reducing the lateral spacing of data points in the $3 \mathrm{D}$ force field to $\sim 0.05 \mathrm{~nm}$.

\section{RESULTS AND DISCUSSION}

Hydration Layer Structure and Dynamics. The equilibrium structure of calcite, dolomite, and magnesite (1014) surfaces and their hydration structure are illustrated in Figure 2. Figure 2a shows ordered hydration layers at the

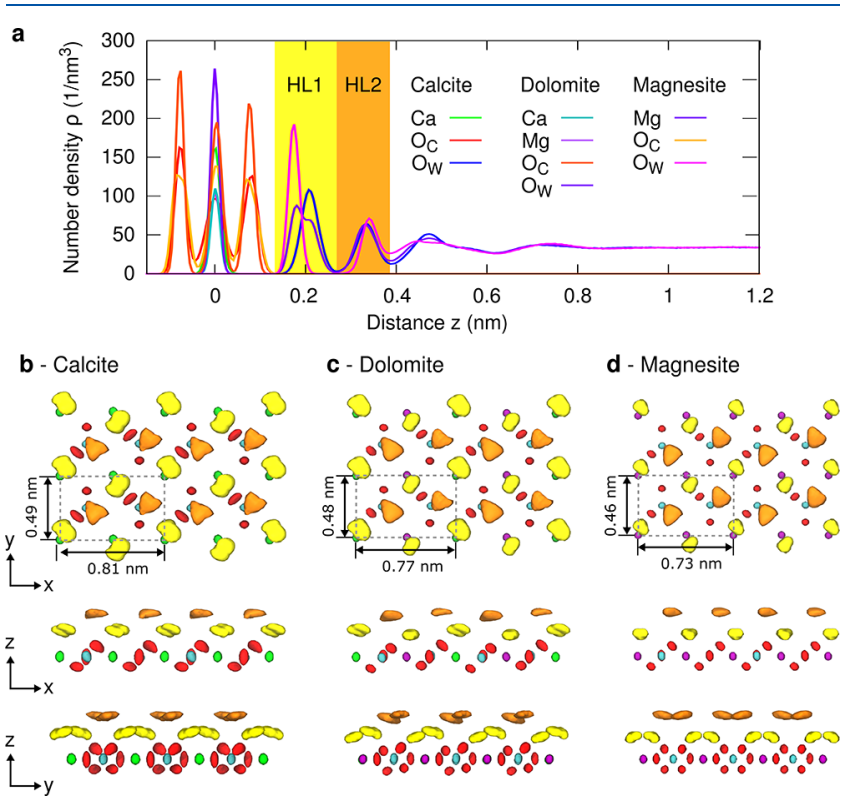

Figure 2. Equilibrium hydration layer structure: number densities of the different species along the direction perpendicular to the calcite-, dolomite-, and magnesite-water interfaces (a). Density isosurface maps of the atoms in the last layer of the surface and the water oxygen atoms in the first (yellow) and second (orange) hydration layers, for calcite (b), dolomite (c), and magnesite (d), in top (xy) and side views $(x z$ and $y z)$. The isosurfaces correspond to four times the density of bulk water.

interface, with the first peak in the water density along the surface normal corresponding to two water molecules in the first hydration layer (HL1) coordinating the two cations in the surface unit cell. The second peak corresponds to a second hydration layer (HL2), consisting of two water molecules situated above the protruding oxygen atoms of the carbonate ions, hydrogen bonded to both the carbonate oxygen and a water molecule in the first hydration layer, as shown in Figure $2 \mathrm{~b}-\mathrm{d}$. Less-ordered third and fourth hydration layers (HL3 and HL4), with lateral density distributions resembling the ones in HL1 and HL2, respectively, can be observed as well, before the water density levels off to the constant value of bulk water. For calcite and magnesite, the water distributions over the two $\mathrm{Ca}$ or $\mathrm{Mg}$ ions in the surface unit cell are symmetric. Dolomite exhibits a bimodal first peak in the water density profile along $z$, because of the water molecules over the Ca and $\mathrm{Mg}$ ions in the surface having different equilibrium positions. In fact, the distributions of the water density over $\mathrm{Ca}$ and $\mathrm{Mg}$ in dolomite are almost identical to the ones over calcite and magnesite, respectively, which can be clearly seen in the density isosurface plots of the water molecule oxygen atoms in HL1 and HL2 shown in Figure $2 b-d$. The peaks corresponding to HL2 and HL3/4 are similar between calcite and dolomite, with magnesite exhibiting an HL2 peak slightly further away from HL1 and slightly less structure in HL3 and HL4. The calcite hydration layers have previously been 
a - Calcite

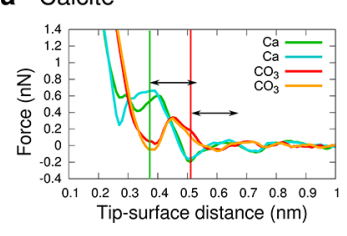

b - Dolomite

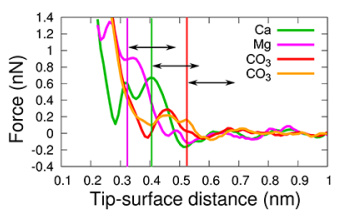

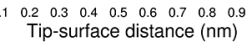

C - Magnesite

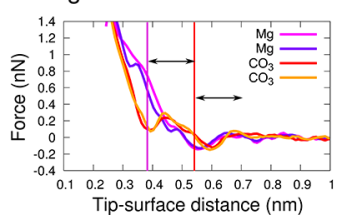

HL1 over Mg

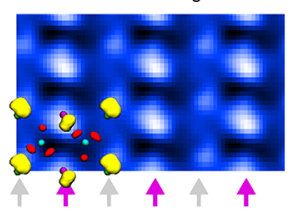

HL1 over Mg

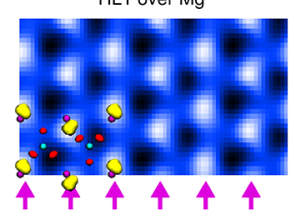

$\mathrm{HL} 2$ over $\mathrm{CO}_{3}$

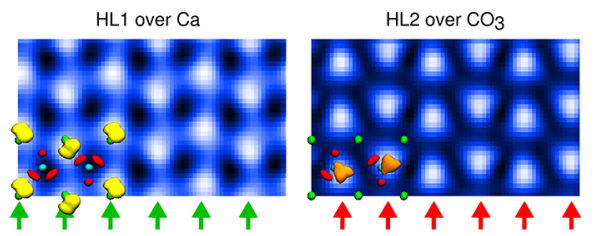

HL1 over $\mathrm{Ca}$
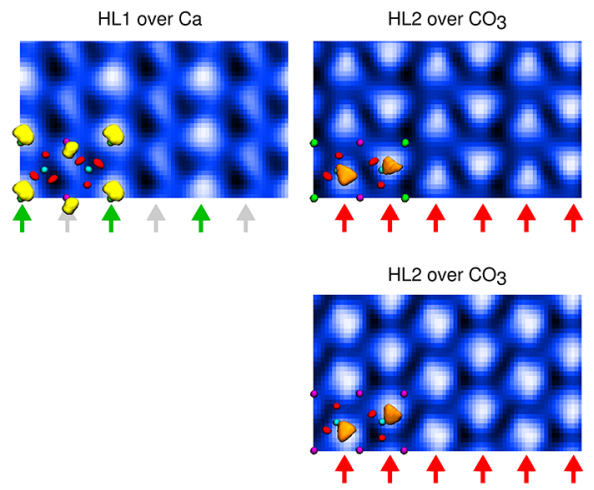

Figure 3. AFM simulation of calcite, dolomite, and magnesite (1014) surfaces in water: (left to right) force-distance curves obtained from free energy calculations with a silica tip model over the two $\mathrm{Ca}$ and/or $\mathrm{Mg}$, and $\mathrm{CO}_{3}$ surface sites and simulated AFM images using the virtual AFM. The images shown were obtained when the lower turning point of the cantilever oscillations was at the $z$ distance indicated by the colored vertical lines, corresponding to the peaks in force associated with the first hydration layer (HL1) over $\mathrm{Mg}$ (magenta lines) and/or Ca (green lines), and the second hydration layer (HL2) over $\mathrm{CO}_{3}$ (red lines), for calcite (a), dolomite (b), and magnesite (c). The tip oscillation ranges, corresponding to $A_{0}$ $=0.08 \mathrm{~nm}$, are indicated by double arrows in the force distance plots. The respective density isosurface maps of the crystal surface and HL1 or HL2 (from Figure 2b) are overlaid on the simulated AFM images to highlight the good agreement between the contrast in the image and the equilibrium hydration layer structure. The positions of the rows of $\mathrm{Mg}, \mathrm{Ca}$, or $\mathrm{CO}_{3}$ ions in the surface underneath the hydration layer that is being imaged at that distance are highlighted by magenta, green, or red arrows, respectively.

investigated by X-ray reflectivity, ${ }^{31}$ and found to be in good agreement with atomistic simulations. ${ }^{32}$

The residence times of first-hydration-layer water molecules were also calculated from MD trajectories using the method reported by De La Pierre et al. ${ }^{33}$ Briefly, we computed the molecules' "survival function"

$$
P(t)=\int_{t}^{\infty} E\left(t^{\prime}\right) \mathrm{d} t^{\prime}
$$

where $E(t) \mathrm{d} t$ represents the probability for a water molecule to remain coordinated to a surface ion site for a time between $t$ and $t+\mathrm{d} t$. This was then fitted with the sum of two exponentially decaying functions

$$
P(t)=\sum_{i=1}^{2} a_{i} \exp \left(-t / \tau_{i}\right)
$$

The time constant $\tau_{1}$ is very small and corresponds to fluctuations in and out of the cut-off distance used to define the HL1 water, whereas $\tau_{2}$ is the actual residence time of the water molecule before an exchange takes place. We find that the time constant for HL1 water over calcite is around $2 \mathrm{~ns}$, as reported earlier, ${ }^{33}$ whereas in dolomite the time constant of water molecules in HL1 over Ca increases to $\tau_{2} \approx 35 \mathrm{~ns}$. The survival functions and exponential fits are shown in Figure S2 in the Supporting Information. Not a single exchange of HL1 water could be observed over the $\mathrm{Mg}$ sites of dolomite and magnesite, over the course of a $200 \mathrm{~ns}$ long MD trajectory. These findings are consistent with the differences in the radial distribution functions of $\mathrm{Ca}$ or $\mathrm{Mg}$ and the oxygen of water $(\mathrm{Ow})$, both for ions in the surface and free ions in solution, as well as water exchange rates on $\mathrm{Mg}^{2+}$ from the experiment. ${ }^{20}$
The hydration layer structure of calcite $(10 \overline{1} 4)$ obtained with the force field of Raiteri et al. ${ }^{22}$ has previously been reported, but this is the first time it has been used to study the hydration layers on dolomite or magnesite. For dolomite, the water density profile obtained in this work resembles the ones obtained by Shen et al. ${ }^{34}$ as well as Söngen et al. ${ }^{18}$ The MD simulations of the dolomite-water interface in ref 18 were carried out using a previous version of the calcite-water potentials by Raiteri et al. ${ }^{35}$ in conjunction with $\mathrm{Mg}$ parameters from Tomono et al. ${ }^{36}$ In this earlier study the Buckingham interactions between $\mathrm{Mg}$ and the carbonate oxygen, as well as the Lennard-Jones parameters for the interaction between $\mathrm{Mg}$ and the water oxygen $(\mathrm{Ow})$ were obtained by rescaling the $\mathrm{Ca}$ parameters reported in ref 37 , rather than by fitting to any physical observables directly. We therefore checked the structural and thermodynamic properties of the $\mathrm{Mg}$ ion in solution using the Tomono parameters: the first and second peaks in the $\mathrm{Mg}-\mathrm{Ow}$ radial distribution function are found at slightly larger distances ( 0.207 and $0.428 \mathrm{~nm}$ vs 0.199 and $0.422 \mathrm{~nm}$, respectively). However, the integral over the radial distribution function yields the same number of six molecules in the first solvation shell. Using free energy perturbation, ${ }^{22}$ the solvation free energy $\Delta G_{\text {solv }}$ of the $\mathrm{Mg}$ ion in SPC/Fw water was calculated to be $-1680 \mathrm{~kJ} \cdot \mathrm{mol}^{-1}$ using the Tomono force field, compared to -1776 and $-1444 \mathrm{~kJ} \cdot \mathrm{mol}^{-1}$ for $\mathrm{Mg}$ and $\mathrm{Ca}$, respectively, using the Raiteri et al. force field, which has been fitted to reproduce experimental values. ${ }^{38}$ As the solvation free energy of $\mathrm{Mg}^{2+}$ is $\sim 100 \mathrm{~kJ} \cdot \mathrm{mol}^{-1}$ too small, both compared to the experimental value and relative to the value for $\mathrm{Ca}$, the Tomono force field underestimates the binding energy and residence time of the water molecule in HL1, as well as the difference in the $z$ position of the HL1 density maxima over Ca and $\mathrm{Mg}$. 
AFM Simulation. The differences in structure and dynamics of water over the $\mathrm{Ca}$ and $\mathrm{Mg}$ sites in calcite, dolomite, and magnesite reported above suggest that an AFM probe, which is sensitive to the equilibrium hydration layer structure, may be able to distinguish between these two sites, as recently argued by Söngen et al. ${ }^{18} \mathrm{~A}$ more stringent test, however, is to perform a simulation of the AFM experiment with an explicit tip model to obtain a 3D force field of the tipsample interaction. The dynamic oscillation of the tip in the 3D force field can then be simulated with a virtual AFM code to obtain simulated AFM images. We have carried out largescale free energy calculations with a model silicon AFM tip on calcite, dolomite, and magnesite. The force-distance curves obtained from the free energy calculations shown in Figure 3 exhibit atomic-scale lateral and vertical resolution, as reported in earlier simulations of AFM imaging of calcite ${ }^{14,39}$ and indicate the possibility to resolve HL1 and HL2 on calcite, dolomite, and magnesite (10 $\overline{1} 4)$ surfaces. The force curves indicate that the interaction of the tip (and its hydration structure) with HL1 is indeed different over the two cation sites in dolomite at distances of $z \leq 0.5 \mathrm{~nm}$. $2 \mathrm{D}$ slices along the $x-z$ plane through the 3D force maps obtained from the AFM simulations, passing through the rows of $\mathrm{Ca} / \mathrm{Mg}$ or $\mathrm{CO}_{3}$ ions, are shown in Figure S3 in the Supporting Information.

In Figure 3a,c, the two curves for $\mathrm{Ca}$ in calcite and $\mathrm{Mg}$ in magnesite correspond to the force curves obtained over the two cation sites in the calcite and magnesite surface unit cell. Whereas the two sites in the respective surface are equivalent by symmetry-both in terms of surface and hydration layer structure-small differences in the force curves can still be noticed. However, these differences between the two equivalent $\mathrm{Ca}$ or $\mathrm{Mg}$ sites are small compared to the difference between the $\mathrm{Ca}$ and $\mathrm{Mg}$ site in the dolomite surface (Figure $3 b)$. We assume that the differences in the two force-distance curves over equivalent surface sites arise predominantly from the asymmetry of the tip apex, whereas the statistical error in the calculation of the free energy profile from which the force curves are derived plays a minor role: the error bars on the free energy profiles from bootstrapping analysis are typically \pm 0.1 to $\pm 0.2 \mathrm{~kJ} \cdot \mathrm{mol}^{-1}$, which is much smaller than the features in the free energy profiles, and also smaller than the differences between the free energy profiles over equivalent sites in the calcite and magnesite surfaces, as shown in Figure S4 in the Supporting Information. We also note that we do not claim that these differences between equivalent sites are related to the elusive "row pairing", first suggested by Jin et al., ${ }^{40}$ based on AFM experiments on calcite (1014) in ambient conditions.

As the differences in the force-distance curves over $\mathrm{Ca}$ and $\mathrm{Mg}$ are quite subtle, it is particularly important to simulate the full cantilever oscillation using a virtual AFM to ensure that the differences in force over the two sites do not get "averaged out" through the oscillation cycle. The PyVAFM code was used to record 2D FM-AFM images at different tip-surface distances over calcite, dolomite, and magnesite surfaces presented in Figure 3. The simulated FM-AFM images, obtained with cantilever oscillation parameters matching the experiments in ref 18 , clearly indicate the possibility to distinguish between the HL1 water molecules over the $\mathrm{Ca}$ and $\mathrm{Mg}$ sites in dolomite. The simulated images also confirm the excellent agreement of the frequency shift map in AFM and the 3D equilibrium structure of the hydration layers for the three systems considered here.
The model silica tip used in the simulation has a sharp termination in a single hydroxyl group, with $\sim 2$ water molecules in the apex hydration shell. Free energy minima, that is vanishing forces, are encountered whenever the hydroxyl oxygen is $\sim 0.27 \mathrm{~nm}$ above the equilibrium position of a surface hydration layer water molecule oxygen. As the tip gets closer, the water molecule can be "trapped" underneath the apex, leading to a repulsive force on the tip, until the force is large enough to trigger a collective re-arrangement of the combined tip and surface hydration structure, coinciding with a marked drop in force. This is a similar imaging mechanism as identified previously for calcite-terminated tips imaging a calcite surface, ${ }^{13}$ even though at larger tip-surface distances the hydration shell around the $\mathrm{OH}$-terminated silica tip can exchange water more readily compared to the Ca-terminated calcite tip model, as shown in the Supporting Information to ref 14. In summary, our simulations strongly support the experimental results of Söngen and co-workers, ${ }^{18}$ and offer a detailed explanation of the origin of contrast that goes beyond the argument given in their paper, based on differences in the equilibrium water structure over the two cation sites.

Water Displacement and Contrast Inversion. A recurring question concerns the AFM tip's ability to displace the HL1 water molecules, and interact directly with one or more atoms in the mineral surface. The three surfaces studied in this work make for interesting test cases, as the surface and hydration layer structures are very similar, but the time scales of water exchange in the first hydration layer vary considerably. In order to explain the contrast formation mechanism in near contact, we have analyzed the configurations of water molecules between tip and $\mathrm{Ca}$ and $\mathrm{Mg}$ ions in the surface of dolomite as a function of the tip-surface distance to explain the changes in the force curves over the two respective HL1 equilibrium positions shown in Figure 4a. We find that for $z>$ $0.30 \mathrm{~nm}$, where the contrast between the $\mathrm{Ca}$ and $\mathrm{Mg}$ sublattice of dolomite is best seen, the first hydration layer remains intact over both sites (Figure $4 \mathrm{~b}$ ). In the range of $0.28 \leq z \leq 0.30$ $\mathrm{nm}$, the AFM tip has effectively displaced the water molecule over $\mathrm{Ca}$, whereas the water molecule over $\mathrm{Mg}$ is still very close to its equilibrium position (Figure $4 \mathrm{c}$ ). The water molecule over $\mathrm{Mg}$ is only displaced when $z \leq 0.24 \mathrm{~nm}$ (Figure $4 \mathrm{~d}$ ). The coinciding drops in force are clearly visible in the forcedistance curves over the $\mathrm{Ca}$ and $\mathrm{Mg}$ sites (Figure 4a). We find that the HL1 water molecule over $\mathrm{Mg}$ is moving toward the equilibrium position of a HL2 water molecule over a neighboring carbonate ion, with the water molecule occupying this position being displaced in turn. This is quite remarkable as the binding of water in the first solvation shell of an $\mathrm{Mg}^{2+}$ ion in solution is much stronger than for $\mathrm{Ca}^{2+}$, and we could not observe any spontaneous exchange of $\mathrm{HL} 1$ water over $\mathrm{Mg}$ in dolomite, or magnesite. Therefore, it is unexpected that the first hydration layer would yield under the load of the tip. A similar analysis shows that the HL1 water over $\mathrm{Ca}$ in calcite is displaced at similar tip-surface distances as over $\mathrm{Ca}$ in dolomite. In contrast, at the distance at which the HL1 water over $\mathrm{Mg}$ in dolomite is displaced, the first hydration layer in magnesite is still intact. However, the maximum forces at these distances are between 1 and $2 \mathrm{nN}$, thus exceeding the usual force threshold in the experiment. It may still prove worthwhile to, quite literally, "push a little harder" to confirm this contrast inversion predicted for a tip apex terminated in a hydroxyl group. The different interaction of a sharp tip with HL1 over various ionic sites also has implications for experimental 
a
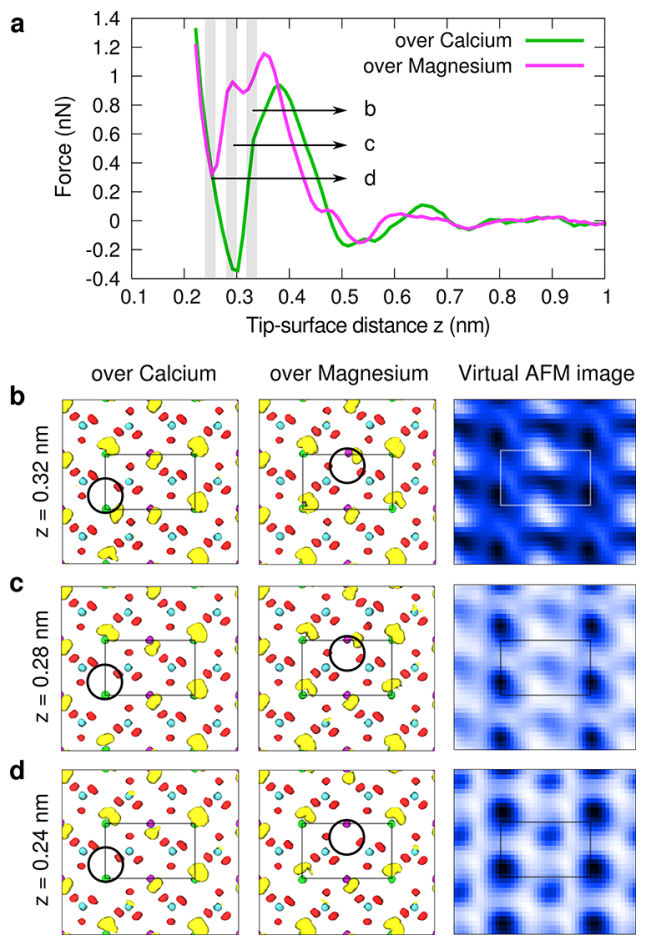

Figure 4. Force-distance curves over the water density maxima in HL1 of dolomite (a). When the tip images HLl over $\mathrm{Mg}$, the water molecule over $\mathrm{Ca}$ is starting to be pushed aside by the tip (b). At closer tip approach, the water molecule over $\mathrm{Ca}$ has been completely removed, whereas the molecule over $\mathrm{Mg}$ is still present (c). At the smallest tip-surface distances simulated, both water molecules in HL1 are displaced (d). The displacement of a water molecule in HL1 can be seen as a drop in the force distance curves, and an inversion of contrast in the virtual AFM image. The lateral position of the AFM tip apex is indicated by a black circle in panels $b-d$.

studies of step growth on crystal surfaces in a supersaturated solution, where increasing slow water exchange rates by the presence of a tip could very well affect the observed growth rates. $^{41}$

\section{CONCLUSIONS}

We have studied the AFM imaging of calcite, dolomite, and magnesite surfaces in water using atomistic $\mathrm{MD}$ and free energy calculations. Although at first glance all three surfaces present very similar hydration layer structures, quantitative analysis reveals subtle differences, with water density distributions over $\mathrm{Ca}$ and $\mathrm{Mg}$ in dolomite closely resembling those over $\mathrm{Ca}$ and $\mathrm{Mg}$ in calcite and magnesite, respectively. However, the time scales for the exchange of water in the first hydration layer were found to differ significantly between the three surfaces, with water exchanging frequently on a nanosecond timescale over calcite, but no exchange over $\mathrm{Mg}$ in dolomite or magnesite could be observed on the time scale typically accessible to atomistic simulation. In addition, we found that water exchange over $\mathrm{Ca}$ in dolomite was slowed down by an order of magnitude compared to calcite. AFM simulations with a model for a silanol-terminated silica tip indicates the possibility to image the hydration layer structures on calcite, dolomite, and magnesite. The difference in force felt by the AFM tip when interacting with water molecules in the first hydration layer over $\mathrm{Ca}$ and $\mathrm{Mg}$ in dolomite is sufficient for lateral contrast between the two sites. This contrast between two divalent cation sites persists even when the full cantilever oscillation is simulated in a virtual AFM experiment. Our results therefore confirm the interpretation by Söngen and co-workers ${ }^{18}$ of their experimental data and strongly support the notion that "chemical sensitivity" between similarly charged surface ions in AFM imaging in solution can be achieved, provided the ions' hydration structures differ sufficiently. Although this has immediate implications for the possibility to image a wide range of mineral surfaces, as well as ionic substitutions in these surfaces with AFM in solution, more general uses of AFM to probe hydration and solvation should also be investigated.

The level of agreement between state of the art experimental AFM data and atomistic AFM simulations in liquid is remarkable, and validates the theoretical approach to approximate forces on the AFM tip by the derivative of the free energy change as a function of the distance between a nanoscale cluster and the surface model, whereby the assumption is made that the tip oscillation can be modeled as a reversible process, and that long-range interactions between the cantilever and the surface are independent of the lateral position of the tip apex. This suggests, on the one hand, the possibility to use the comparison between hydration layer structure observed in simulation with AFM experiments directly to fine-tune force field interaction parameters in the simulations. On the other hand, the remaining small quantitative differences highlight the fact that the limiting factor in the predictive power of simulations is the unknown atomistic structure of the tip apex, and a need to systematically investigate different possible tip terminations for commonly used silicon or silicon nitride AFM tips in water, as well as their interactions with ions typically present in proximity of the solid-liquid interface.

\section{ASSOCIATED CONTENT}

\section{S Supporting Information}

The Supporting Information is available free of charge on the ACS Publications website at DOI: 10.1021/acs.jpcc.9b00939.

Construction and validation of the tip-surface interaction parameters, survival functions and time constants for water exchange over $\mathrm{Ca}$ in calcite and dolomite, $2 \mathrm{D}$ slices along the $x-z$ plane through the $3 \mathrm{D}$ force maps obtained from the AFM simulation, as well as selected free energy profiles from umbrella sampling with error bars from bootstrapping analysis (PDF)

\section{AUTHOR INFORMATION}

\section{Corresponding Author}

*E-mail: bernhard.reischl@helsinki.fi. Phone: +358 50 3768922.

ORCID $\odot$

Bernhard Reischl: 0000-0001-7333-4923

Paolo Raiteri: 0000-0003-0692-0505

Andrew L. Rohl: 0000-0003-0038-2785

Notes

The authors declare no competing financial interest.

\section{ACKNOWLEDGMENTS}

This work has been supported by the Australian Research Council Discovery Project DP140101776 and Future Fellowship FT130100463 to P.R. The computational resources have 
been provided by the Australian Government and the Government of Western Australia through the Pawsey Supercomputing Centre under the National Computational Merit Allocation Scheme.

\section{REFERENCES}

(1) Fukuma, T.; Kobayashi, K.; Matsushige, K.; Yamada, H. True Atomic Resolution in Liquid by Frequency-Modulation Atomic Force Microscopy. Appl. Phys. Lett. 2005, 87, 034101.

(2) Rode, S.; Oyabu, N.; Kobayashi, K.; Yamada, H.; Kühnle, A. True Atomic-Resolution Imaging of (1014) Calcite in Aqueous Solution by Frequency Modulation Atomic Force Microscopy. Langmuir 2009, 25, 2850-2853.

(3) Imada, H.; Kimura, K.; Onishi, H. Atom-Resolved AFM Imaging of Calcite Nanoparticles in Water. Chem. Phys. 2013, 419, 193-195.

(4) Fukuma, T. Water Distribution at Solid/Liquid Interfaces Visualized by Frequency Modulation Atomic Force Microscopy. Sci. Technol. Adv. Mater. 2010, 11, 033003.

(5) Kobayashi, K.; Oyabu, N.; Kimura, K.; Ido, S.; Suzuki, K.; Imai, T.; Tagami, K.; Tsukada, M.; Yamada, H. Visualization of Hydration Layers on Muscovite Mica in Aqueous Solution by FrequencyModulation Atomic Force Microscopy. J. Chem. Phys. 2013, 138, 184704.

(6) Marutschke, C.; Walters, D.; Cleveland, J.; Hermes, I.; Bechstein, R.; Kühnle, A. Three-Dimensional Hydration Layer Mapping on the (10.4) Surface of Calcite Using Amplitude Modulation Atomic Force Microscopy. Nanotechnology 2014, 25, 335703.

(7) Martin-Jimenez, D.; Chacon, E.; Tarazona, P.; Garcia, R. Atomically Resolved Three-Dimensional Structures of Electrolyte Aqueous Solutions Near a Solid Surface. Nat. Commun. 2016, 7, 12164.

(8) Watkins, M.; Shluger, A. Mechanism of Contrast Formation in Atomic Force Microscopy in Water. Phys. Rev. Lett. 2010, 105, 196101.

(9) Watkins, M.; Berkowitz, M. L.; Shluger, A. L. Role of Water in Atomic Resolution AFM in Solutions. Phys. Chem. Chem. Phys. 2011, 13, 12584 .

(10) Argyris, D.; Phan, A.; Striolo, A.; Ashby, P. D. Hydration Structure at the $\alpha-\mathrm{Al}_{2} \mathrm{O}_{3}(0001)$ Surface: Insights from Experimental Atomic Force Spectroscopic Data and Atomistic Molecular Dynamics Simulations. J. Phys. Chem. C 2013, 117, 10433-10444.

(11) Watkins, M.; Reischl, B. A Simple Approximation for Forces Exerted on an AFM Tip in Liquid. J. Chem. Phys. 2013, 138, 154703.

(12) Harada, M.; Tsukada, M. Tip-Sample Interaction Force Mediated by Water Molecules for AFM in Water: Three-Dimensional Reference Interaction Site Model Theory. Phys. Rev. B: Condens. Matter Mater. Phys. 2010, 82, 035414.

(13) Fukuma, T.; Reischl, B.; Kobayashi, N.; Spijker, P.; Canova, F. F.; Miyazawa, K.; Foster, A. S. Mechanism of Atomic Force Microscopy Imaging of Three-Dimensional Hydration Structures at a Solid-Liquid Interface. Phys. Rev. B: Condens. Matter Mater. Phys. 2015, 92, 155412.

(14) Reischl, B.; Raiteri, P.; Gale, J. D.; Rohl, A. L. Can Point Defects in Surfaces in Solution be Atomically Resolved by Atomic Force Microscopy? Phys. Rev. Lett. 2016, 117, 226101.

(15) Söngen, H.; Reischl, B.; Miyata, K.; Bechstein, R.; Raiteri, P.; Rohl, A. L.; Gale, J. D.; Fukuma, T.; Kühnle, A. Resolving Point Defects in the Hydration Structure of Calcite (10.4) with ThreeDimensional Atomic Force Microscopy. Phys. Rev. Lett. 2018, 120, 116101.

(16) Kawai, S.; Pina, C. M.; Bubendorf, A.; Fessler, G.; Glatzel, T.; Gnecco, E.; Meyer, E. Systematic Study of the Dolomite (104) Surface by Bimodal Dynamic Force Microscopy in Ultra-High Vacuum. Nanotechnology 2013, 24, 055702.

(17) Pina, C. M.; Pimentel, C.; García-Merino, M. High Resolution Imaging of the Dolomite (104) Cleavage Surface by Atomic Force Microscopy. Surf. Sci. 2010, 604, 1877-1881.
(18) Söngen, H.; Marutschke, C.; Spijker, P.; Holmgren, E.; Hermes, I.; Bechstein, R.; Klassen, S.; Tracey, J.; Foster, A. S.; Kühnle, A. Chemical Identification at the Solid-Liquid Interface. Langmuir 2017, $33,125-129$.

(19) Plimpton, S. Fast Parallel Algorithms for Short-Range Molecular Dynamics. J. Comput. Phys. 1995, 117, 1-19.

(20) Bleuzen, A.; Pittet, P.-A.; Helm, L.; Merbach, A. E. Water Exchange on Magnesium (II) in Aqueous Solution: a Variable Temperature and Pressure ${ }^{17} \mathrm{O}$ NMR Study. Magn. Reson. Chem. 1997, 35, 765-773.

(21) Allnér, O.; Nilsson, L.; Villa, A. Magnesium Ion-Water Coordination and Exchange in Biomolecular Simulations. J. Chem. Theory Comput. 2012, 8, 1493-1502.

(22) Raiteri, P.; Demichelis, R.; Gale, J. D. Thermodynamically Consistent Force Field for Molecular Dynamics Simulations of Alkaline-Earth Carbonates and Their Aqueous Speciation. J. Phys. Chem. C 2015, 119, 24447-24458.

(23) Wu, Y.; Tepper, H. L.; Voth, G. A. Flexible Simple PointCharge Water Model with Improved Liquid-State Properties. J. Chem. Phys. 2006, 124, 024503.

(24) Cygan, R. T.; Liang, J.-J.; Kalinichev, A. G. Molecular Models of Hydroxide, Oxyhydroxide, and Clay Phases and the Development of a General Force Field. J. Phys. Chem. B 2004, 108, 1255-1266.

(25) Skelton, A. A.; Fenter, P.; Kubicki, J. D.; Wesolowski, D. J.; Cummings, P. T. Simulations of the Quartz $(10 \overline{1} 1) /$ Water Interface: A Comparison of Classical Force Fields, Ab Initio Molecular Dynamics, and X-ray Reflectivity Experiments. J. Phys. Chem. C 2011, 115, 2076-2088.

(26) Skelton, A. A.; Wesolowski, D. J.; Cummings, P. T. Investigating the Quartz $(10 \overline{1} 0) /$ Water Interface using Classical and Ab Initio Molecular Dynamics. Langmuir 2011, 27, 8700-8709.

(27) Reischl, B.; Watkins, M.; Foster, A. S. Free Energy Approaches for Modeling Atomic Force Microscopy in Liquids. J. Chem. Theory Comput. 2013, 9, 600-608.

(28) Tribello, G. A.; Bonomi, M.; Branduardi, D.; Camilloni, C.; Bussi, G. Plumed 2: New Feathers for an Old Bird. Comput. Phys. Commun. 2014, 185, 604-613.

(29) Grossfield, A. An Implementation of WHAM: The Weighted Histogram Analysis Method. http://membrane.urmc.rochester.edu (accessed May 22, 2019).

(30) Tracey, J.; Federici Canova, F.; Keisanen, O.; Gao, D. Z.; Spijker, P.; Reischl, B.; Foster, A. S. Flexible and Modular Virtual Scanning Probe Microscope. Comput. Phys. Commun. 2015, 196, $429-438$.

(31) Fenter, P.; Sturchio, N. C. Calcite (104)-Water Interface Structure, Revisited. Geochim. Cosmochim. Acta 2012, 97, 58-69.

(32) Fenter, P.; Kerisit, S.; Raiteri, P.; Gale, J. D. Is the CalciteWater Interface Understood? Direct Comparisons of Molecular Dynamics Simulations with Specular X-ray Reflectivity Data. J. Phys. Chem. C 2013, 117, 5028-5042.

(33) De La Pierre, M.; Raiteri, P.; Gale, J. D. Structure and Dynamics of Water at Step Edges on the Calcite $\{10 \overline{1} 4\}$ Surface. Cryst. Growth Des. 2016, 16, 5907-5914.

(34) Shen, Z.; Szlufarska, I.; Brown, P. E.; Xu, H. Investigation of the Role of Polysaccharide in the Dolomite Growth at Low Temperature by Using Atomistic Simulations. Langmuir 2015, 31, 10435-10442.

(35) Raiteri, P.; Gale, J. D. Water Is the Key to Nonclassical Nucleation of Amorphous Calcium Carbonate. J. Am. Chem. Soc. 2010, 132, 17623-17634.

(36) Tomono, H.; Nada, H.; Zhu, F.; Sakamoto, T.; Nishimura, T.; Kato, T. Effects of Magnesium Ions and Water Molecules on the Structure of Amorphous Calcium Carbonate: A Molecular Dynamics Study. J. Phys. Chem. B 2013, 117, 14849-14856.

(37) Raiteri, P.; Gale, J. D.; Quigley, D.; Rodger, P. M. Derivation of an Accurate Force-Field for Simulating the Growth of Calcium Carbonate from Aqueous Solution: A New Model for the CalciteWater Interface. J. Phys. Chem. C 2010, 114, 5997-6010. 
(38) David, F.; Vokhmin, V.; Ionova, G. Water Characteristics Depend on the Ionic Environment. Thermodynamics and Modelisation of the Aquo Ions. J. Mol. Liq. 2001, 90, 45-62.

(39) Tracey, J.; Miyazawa, K.; Spijker, P.; Miyata, K.; Reischl, B.; Canova, F. F.; Rohl, A. L.; Fukuma, T.; Foster, A. S. Understanding 2D Atomic Resolution Imaging of the Calcite Surface in Water by Frequency Modulation Atomic Force Microscopy. Nanotechnology 2016, 27, 415709.

(40) Jin, M.; Shimada, E.; Ikuma, Y. Observation of Calcite (1014) Surface by AFM in Air and Surface Structure Analysis. J. Ceram. Soc. Jpn. 1999, 107, 1166-1170.

(41) Bracco, J. N.; Stack, A. G.; Higgins, S. R. Magnesite Step Growth Rates as a Function of the Aqueous Magnesium:Carbonate Ratio. Cryst. Growth Des. 2014, 14, 6033-6040. 